\title{
Termodinâmica Estendida para Materiais Viscoelásticos com Condução de Calor
}

\author{
A. VIGNATTI ${ }^{1}$, Departamento de Engenharia e Ciências Exatas da UFES, \\ 29933-480 São Mateus, ES, Brasil.
}

I-SHIH LIU², Instituto de Matemática da UFRJ, 21945-970 Rio de Janeiro, RJ, Brasil.

Resumo. Nesse artigo será apresentada uma formulação de termodinâmica estendida para materiais viscoelásticos com condução de calor. O requerimento da invariância Galileana sobre as equações de balanço e o princípio de entropia conduzem à introdução de multiplicadores de Lagrange, que estabelecem equações constitutivas para os fluxos. Uma condição de hiperbolicidade do sistema de equações é obtido por meio da concavidade da densidade de entropia.

\section{Introdução}

A termodinâmica estendida é uma teoria que complementa as leis usuais de conservação de massa, momento e energia, empregadas na termodinâmica clássica, com um conjunto de equações de balanço adicionais que envolvem o fluxo de calor. Em um total de 13 equações de balanço são determinados 13 momentos: densidade, velocidade, tensor tensão e fluxo de calor [9], [12].

A termodinâmica estendida tem sido aplicada a gases [3], [11], [12], assim como fluidos [10], [13] e sólidos viscoelásticos [6],[7],[8]. Em particular, Vignatti e Liu [13] consideraram a energia como um campo independente e aumentaram a equação da energia total, resultando em um sistema de 14 equações de balanço. O requerimento da invariância Galileana sobre o sistema de equações implica na decomposição dos momentos e fluxos como produtos de duas funções em que uma delas independe do campo velocidade [9], [15].

Este artigo dá continuidade ao trabalho apresentado em [13], seguindo o procedimento nas referências ali citadas. A diferença significativa do artigo [13] para este está na inclusão da equação (3.2), o que caracteriza a teoria para sólidos. Admitindo a concavidade da densidade entropia, foi possível mostrar a positividade do calor específico, a volume constante.

Será usada a mesma notação indicial utilizada em [13]. Em particular $A_{(i j)}=$ $\frac{1}{2}\left(A_{i j}+A_{j i}\right)$ e o símbolo \langle\rangle indica a simetrização sem traço $A_{\langle i j\rangle}=A_{(i j)}-\frac{1}{3} A_{s s} \delta_{i j}$.

\footnotetext{
${ }^{1}$ aldovignatti@ceunes.ufes.br

${ }^{2}$ liu@im.ufrj.br
} 


\section{Equações de Balanço}

As leis de conservação de massa, momento e energia em um sistema de coordenadas espaciais $\left(x_{i}, t\right)$ relativo a um referencial inercial, podem ser escritas como

$$
\begin{gathered}
\frac{\partial \varrho}{\partial t}+\frac{\partial \varrho v_{k}}{\partial x_{k}}=0 \\
\frac{\partial \varrho v_{i}}{\partial t}+\frac{\partial}{\partial x_{k}}\left(\varrho v_{i} v_{k}-T_{i k}\right)=0, \\
\frac{\partial \varrho e}{\partial t}+\frac{\partial}{\partial x_{k}}\left(\varrho e v_{k}-v_{i} T_{i k}+q_{k}\right)=0,
\end{gathered}
$$

onde $\varrho$ é a densidade de massa, $v_{i}$ a velocidade, $T_{i k}$ o tensor tensão de Cauchy, $q_{k}$ o fluxo de energia, $e=\left(v^{2} / 2+\varepsilon\right)$ é a energia total específica e $\varepsilon$ a energia interna específica.

A fim de obter um modelo matemático hiperbólico para materiais viscoelásticos com condução de calor são acrescentadas as equações de balanço

$$
\begin{gathered}
\frac{\partial u_{i j}}{\partial t}+\frac{\partial}{\partial x_{k}}\left(u_{i j} v_{k}+G_{i j k}\right)=P_{i j}, \\
\frac{\partial u_{i i j}}{\partial t}+\frac{\partial}{\partial x_{k}}\left(u_{i i j} v_{k}+G_{i i j k}\right)=P_{i i j},
\end{gathered}
$$

para os momentos $u_{i j}, u_{i i j}$, seus fluxos $G_{i j k}, G_{i i j k}$ e produções $P_{i j}$ e $P_{i i j}$. Estas equações são acrescentadas para ser construída uma teoria estendida de 14 momentos para materiais viscoelásticos com condução de calor, por exemplo sólidos. Ao contrário das teorias ordinárias a inclusão das equações (2.2) para $u_{i j}$ e $u_{i i j}$ são particulares da teoria estendida. Elas não são leis de conservação devido à presença dos termos de produção $P_{i j}$ e $P_{i i j}$ no membro direito.

Analogamente a [13], as partes independentes da velocidade, conhecidas como partes internas dos momentos $u_{i j}$ e $u_{i i j}$ serão denotadas por $\varrho_{i j}$ e $\varrho_{i i j}$, dos fluxos $G_{i j k}$ e $G_{i i j k}$ por $p_{i j k}$ e $p_{i i j k}$ das produções $P_{i j}$ e $P_{i i j}$ por $\pi_{i j}$ e $\pi_{i i j}$, respectivamente.

Motivado pela teoria cinética de gases, os termos $u_{i j}, G_{i j k}, P_{i j}, u_{i i j}, G_{i i j k}, P_{i i j}$ serão admitidos simétricos nos índices $i, j$. Será admitido ainda que $\pi_{0}=0, \pi_{i}=0$ e que as quantidades $\varrho_{i j}, p_{i j}=-T_{i j}, p_{i j k}, \pi_{i j}, p_{i i k}, p_{i i j k}, \pi_{i i j}$ assim como $\varrho, \varepsilon, T_{i k}$, $q_{k}$ são quantidades objetivas.

O requerimento da invariância Galileana implica na dependência explícita da velocidade $v_{i}[15]$ (para mais detalhes, ver [12]), e portanto o sistema (2.1) e (2.2) pode ser escrito no sistema de coordenadas materiais como 


$$
\begin{aligned}
\dot{\varrho}_{\kappa} & =0, \\
\varrho_{\kappa} \dot{v}_{i}+\frac{\partial \hat{p}_{i \alpha}}{\partial X_{\alpha}} & =0, \\
\varrho_{\kappa} \dot{e}+\frac{\partial \hat{G}_{\alpha}}{\partial X_{\alpha}} & =0, \\
\dot{\hat{u}}_{i j}+\frac{\partial \hat{G}_{i j \alpha}}{\partial X_{\alpha}} & =\varrho_{\kappa} \pi_{i j}, \\
\dot{\hat{u}}_{i i j}+\frac{\partial \hat{G}_{i i j \alpha}}{\partial X_{\alpha}} & =\varrho_{\kappa} \pi_{i i j}+3 \varrho_{\kappa} v_{(i} \pi_{i j)},
\end{aligned}
$$

onde

$$
\begin{aligned}
\hat{u}_{i j} & =\varrho_{\kappa} \varrho_{i j}+\varrho_{\kappa} v_{i} v_{j}, \\
\hat{u}_{i i j} & =\varrho_{\kappa} \varrho_{i i j}+3 \varrho_{\kappa} \varrho_{(i i} v_{j)}+\varrho_{\kappa} v_{i} v_{i} v_{j}, \\
\hat{G}_{\alpha} & =\hat{q}_{\alpha}+v_{i} \hat{p}_{i \alpha} \\
\hat{G}_{i j \alpha} & =\hat{p}_{i j \alpha}+v_{i} \hat{p}_{j \alpha}+v_{j} \hat{p}_{i \alpha}, \\
\hat{G}_{i i j k} & =\hat{p}_{i i j k}+3 v_{(i} v_{i} \hat{p}_{j) k}+3 v_{(i} \hat{p}_{i j) k},
\end{aligned}
$$

O sistema (2.3) é equivalente ao sistema de equações de balanço consistindo de (2.1) e (2.2). O tensor $\hat{T}_{i \alpha}=-\hat{p}_{i \alpha}$ é o tensor tensão Piola-Kirchoff e $\hat{q}_{\alpha}$ é algumas vezes chamado por fluxo de energia material.

Para materiais viscoelásticos, um estado pode ser caracterizado pelos seguintes campos termodinâmicos:

$$
\begin{aligned}
& F_{i \alpha} \text { gradiente de deformação, } \\
& v_{i} \text { velocidade, } \\
& \varepsilon \text { energia interna específica, } \\
& T_{i j} \text { tensor tensão, } \\
& q_{j} \text { fluxo de calor. }
\end{aligned}
$$

Obviamente, o gradiente de deformação está sendo considerado no lugar da densidade, como um campo termodinâmico para que os sólidos possam ser incluídos na teoria.

O sistema (2.3) deve ser completado por relações constitutivas que em termodinâmicas estendidas são assumidas serem locais e instantâneas.

Equações constitutivas não são inteiramente arbitrárias. Elas estão restritas por um princípio físico universal e, em particular o princípio de entropia e objetividade material. Mais a frente será imposta uma condição de hiperbolicidade.

\section{Princípio de Entropia}

O princípio de entropia estabelece que para todo processo termodinâmico a inequação de entropia deve ser válida

$$
\varrho_{\kappa} \dot{\eta}+\frac{\partial \hat{\Phi}_{\alpha}}{\partial X_{\alpha}}=s \geq 0 .
$$


Esta também está escrita no sistema de coordenadas materiais e similarmente é introduzido $\hat{\Phi}_{\alpha}=\left(\varrho_{\kappa} / \varrho\right) \Phi_{\kappa} F_{\alpha k}^{-1}$, onde $\Phi_{\kappa}$ é o fluxo de entropia. Além disso, a função $\eta$ é admitida ser côncava nas variáveis-campo básicas e tanto $\eta$ quanto $\Phi=\left(\Phi_{1}, \Phi_{2}, \Phi_{3}\right)$ são admitidas ser objetivas. A produção de entropia $s$ é uma quantidade não negativa.

Observe que os campos $F_{s \alpha}$ e $v_{s}$ não são inteiramente independentes. De acordo com suas definições, eles estão relacionados pela seguinte identidade

$$
\dot{F}_{s \alpha}-\frac{\partial v_{s}}{\partial X_{\alpha}}=0
$$

Observe ainda que a primeira equação de $(2.3), \dot{\varrho}_{\kappa}=0$, meramente afirma que $\varrho_{\kappa}$ é um campo independente do tempo. Juntando as outras equações de (2.3) com (3.2) obtém-se um sistema que pode ser escrito na forma

$$
A_{a b} Y_{b}+B_{a}=0 .
$$

Isto e a objetividade de $\eta$ e $\hat{\Phi}_{\alpha}$ implicam na existência de multiplicadores de Lagrange [5] $\lambda_{i \beta}, \Lambda, \Lambda_{i j}, \lambda_{j}, \Lambda_{i}$ tais que

$$
\begin{aligned}
d \eta & =\lambda_{i \beta} d F_{i \beta}+\Lambda d \varepsilon+\Lambda_{r l} d \varrho_{r l}+\lambda_{l} d \varrho_{r r l}, \\
0 & =\Lambda_{i}+3 \lambda_{(i} \varrho_{r r)}, \\
d \hat{\Phi}_{\alpha} & =\Lambda d \hat{q}_{\alpha}+\Lambda_{i} d \hat{p}_{i \alpha}+\Lambda_{i j} d \hat{p}_{i j \alpha}+\lambda_{j} d \hat{p}_{i i j \alpha}, \\
0 & =-\varrho_{\kappa} \lambda_{i \alpha}+\Lambda \hat{p}_{i \alpha}+2 \Lambda_{i j} \hat{p}_{j \alpha}+3 \lambda_{(j} \hat{p}_{i j) \alpha} \\
s & =\varrho_{\kappa}\left(\Lambda_{r l} \pi_{r l}+\lambda_{l} \pi_{r r l}\right) \geq 0 .
\end{aligned}
$$

\section{Equilíbrio}

O equilíbrio é definido como um processo termodinâmico sem produção de entropia. De acordo com [13], temos que a condição necessária para o mínimo da produção de entropia implica que

$$
0=\left.\frac{\partial s}{\partial \pi_{i j}}\right|_{E}=\left.\varrho_{\kappa} \Lambda_{i j}\right|_{E}, \quad 0=\left.\frac{\partial s}{\partial \pi_{i i j}}\right|_{E}=\left.\varrho_{\kappa} \lambda_{j}\right|_{E} .
$$

Tanto $\left.\right|_{E}$ quanto o índice $o$ denotarão a avaliação no equilíbrio. De $(3.3)_{2}$ tem-se $\left.\Lambda_{k}\right|_{E}=0$.

Avalie a relação $(3.3)_{1}$ no equilíbrio e use $(3.3)_{4}$ para ter

$$
\left.d \eta\right|_{E}=\left.\lambda_{i \beta}\right|_{E} d F_{i \beta}+\left.\Lambda\right|_{E} d \varepsilon=\left.\Lambda\right|_{E}\left(d \varepsilon+\frac{1}{\varrho_{\kappa}} \hat{p}_{i \beta}^{o} d F_{i \beta}\right) .
$$

Comparando com a relação de Gibbs[14]

$$
d \eta_{o}=\frac{1}{\theta}\left(d \varepsilon+\frac{1}{\varrho_{\kappa}} \hat{p}_{i \beta}^{o} d F_{i \beta}\right),
$$

para materiais elásticos, decorrente da teoria ordinária [9], tem-se as identificações

$$
\left.\Lambda\right|_{E}=\frac{1}{\theta},
$$


onde $\theta$ é a temperatura. Por $(3.3)_{4}$

$$
\left.\lambda_{i \beta}\right|_{E}=\frac{1}{\theta \varrho_{\kappa}} \hat{p}_{i \beta}^{o} .
$$

Cada um dos termos, definidos abaixo,

$$
\begin{array}{ll}
\Lambda_{\varepsilon}=\Lambda-\frac{1}{\theta}, & \Lambda_{i \beta}^{\varepsilon}=\lambda_{i \beta}-\frac{1}{\theta \varrho_{\kappa}} \hat{p}_{i \beta}^{o}, \\
\hat{S}_{i j}=\hat{p}_{i j}^{o}-\hat{p}_{i j}, & \varrho_{\varepsilon}=\varrho_{s s}-\left.\varrho_{s s}\right|_{E},
\end{array}
$$

se anula no equilíbrio e $\Lambda_{\varepsilon}, \Lambda_{i \beta}^{\varepsilon}$ herdarão o nome de multiplicadores de Lagrange.

Analogamente a [13], a função

$$
\hat{\eta}=\Lambda_{\langle i j\rangle} \varrho_{\langle i j\rangle}+\lambda_{j} \varrho_{i i j}-\eta,
$$

e a conjugada do fluxo de entropia $\hat{\Phi}_{k}$

$$
\check{\Phi}_{k}=\Lambda \hat{q}_{k}+\Lambda_{i} \hat{p}_{i k}+\Lambda_{i j} \hat{p}_{i j k}+\lambda_{j} \hat{p}_{i i j k}-\hat{\Phi}_{k},
$$

satisfazem

$$
\begin{aligned}
d \hat{\eta}= & \left(\Lambda_{i \beta}^{\varepsilon}+\Lambda_{\varepsilon} \frac{\partial \varepsilon}{\partial F_{i \beta}}+\frac{\partial \eta_{0}}{\partial F_{i \beta}}+\frac{1}{3} \Lambda_{r r} \frac{\left.\partial \varrho_{s s}\right|_{E}}{\partial F_{i \beta}}\right) d F_{i \beta} \\
& +\left(\Lambda_{\varepsilon} \frac{\partial \varepsilon}{\partial \theta}+\frac{\partial \eta_{0}}{\partial \theta}+\frac{1}{3} \Lambda_{r r} \frac{\left.\partial \varrho_{s s}\right|_{E}}{\partial \theta}\right) d \theta+\frac{1}{3} \Lambda_{r r} d \varrho_{\varepsilon}+\varrho_{\langle i j\rangle} d \Lambda_{\langle i j\rangle}+\varrho_{i i j} d \lambda_{j}, \\
d \check{\Phi}_{k}= & -\frac{1}{\theta^{2}} \hat{q}_{k} d \theta+\hat{q}_{k} d \Lambda_{\varepsilon}+\hat{p}_{i k} d \Lambda_{i}+\hat{p}_{\langle i j\rangle k} d \Lambda_{\langle i j\rangle}+\frac{1}{3} \hat{p}_{i i k} d \Lambda_{j j}+\hat{p}_{i i j k} d \lambda_{j} .
\end{aligned}
$$

e assumindo que $\varrho_{\varepsilon}, \Lambda_{\varepsilon}, \Lambda_{i}, \Lambda_{\langle i j\rangle}, \Lambda_{j j}$ e $\lambda_{j}$ são quantidades de ordem 1, obtem-se (veja [9]),

$$
\begin{aligned}
\hat{\eta}= & \eta_{0}+k_{0} \varrho_{\varepsilon}+h_{1} \lambda_{j} \lambda_{j}+h_{2} \Lambda_{\langle i j\rangle} \Lambda_{\langle i j\rangle}+h_{3} \varrho_{\varepsilon}^{2}+o(3), \\
\check{\Phi}_{k}= & \alpha \lambda_{k}+\beta \Lambda_{k}+a_{1} \Lambda_{\varepsilon} \lambda_{k}+b_{1} \Lambda_{\varepsilon} \Lambda_{k}+a_{2} \Lambda_{\langle k i\rangle} \lambda_{i}+b_{2} \Lambda_{\langle k i\rangle} \Lambda_{i} \\
& +a_{3} \Lambda_{i i} \lambda_{k}+b_{3} \Lambda_{i i} \Lambda_{k}+o(3),
\end{aligned}
$$

onde os coeficientes $\eta_{0}, k_{0}, h_{n}, \alpha, \beta, a_{n}, b_{n}$, são funções de $\left(F_{i \beta}, \theta\right)$. A notação $o(n)$ representa termos de ordem maior ou igual a $n$ nas quantidades $\Lambda_{\varepsilon}, \Lambda_{i}, \Lambda_{\langle i j\rangle}, \Lambda_{j j}$ e $\lambda_{j}$. 


\section{Equações Constitutivas de Primeira Ordem}

Comparando (4.9) com (4.10) ${ }_{1}$ obtem-se as expressões

$$
\begin{aligned}
\varrho_{i i j} & =2 h_{1} \lambda_{j}+o(2), \\
\frac{1}{3} \Lambda_{r r} & =k_{0}+2 h_{3} \varrho_{\varepsilon}+o(2), \\
\varrho_{\langle i j\rangle} & =2 h_{2} \Lambda_{\langle i j\rangle}+o(2), \\
\Lambda_{\varepsilon} \frac{\partial \varepsilon}{\partial \theta}+\frac{1}{3} \Lambda_{r r} \frac{\left.\partial \varrho_{s s}\right|_{E}}{\partial \theta} & =\frac{\partial k_{0}}{\partial \theta} \varrho_{\varepsilon}+o(2), \\
\Lambda_{i \beta}^{\varepsilon}+\Lambda_{\varepsilon} \frac{\partial \varepsilon}{\partial F_{i \beta}}+\frac{1}{3} \Lambda_{r r} \frac{\left.\partial \varrho_{s s}\right|_{E}}{\partial F_{i \beta}} & =\frac{\partial k_{0}}{\partial F_{i \beta}} \varrho_{\varepsilon}+o(2),
\end{aligned}
$$

para os multiplicadores de Lagrange. A equação $(5.1)_{2}$ avaliada no equilíbrio revela que $k_{0}=0$, e como conseqüência

$$
\begin{aligned}
\Lambda_{\varepsilon} & =-2 c_{\theta} h_{3} \varrho_{\varepsilon}+o(2) \\
\Lambda_{i \beta}^{\varepsilon} & =\left(\frac{\partial \varepsilon}{\partial F_{i \beta}} c_{\theta}-\frac{\left.\partial \varrho_{s s}\right|_{E}}{\partial F_{i \beta}}\right) 2 h_{3} \varrho_{\varepsilon}+o(2),
\end{aligned}
$$

onde, $c_{\theta}=\left(\frac{\partial \varepsilon}{\partial \theta}\right)^{-1} \frac{\left.\partial \varrho_{s s}\right|_{E}}{\partial \theta}$. Usando $(3.3)_{2}$ encontra-se

$$
\Lambda_{i}=-\frac{5}{6} \frac{\left.\varrho_{s s}\right|_{E}}{h_{1}} \varrho_{j j i}+o(2) .
$$

Comparando (4.9) com $(4.10)_{2}$ obtem-se as expressões

$$
\begin{aligned}
-\frac{1}{\theta^{2}} \hat{q}_{k} & =\frac{\partial \alpha}{\partial \theta} \lambda_{k}+\frac{\partial \beta}{\partial \theta} \Lambda_{k}+o(2), \\
\hat{q}_{k} & =a_{1} \lambda_{k}+b_{1} \Lambda_{k}+o(2), \\
\hat{p}_{i k} & =\beta \delta_{i k}+b_{1} \Lambda_{\varepsilon} \delta_{i k}+b_{2} \Lambda_{\langle k i\rangle}+b_{3} \Lambda_{j j} \delta_{i k}+o(2), \\
\hat{p}_{\langle i j\rangle k} & =a_{2} \delta_{k\langle i} \lambda_{j\rangle}+b_{2} \delta_{k\langle i} \Lambda_{j\rangle}+o(2), \\
\frac{1}{3} \hat{p}_{i i k} & =a_{3} \lambda_{k}+b_{3} \Lambda_{k}+o(2), \\
\hat{p}_{i i j k} & =\left(\alpha+a_{1} \Lambda_{\varepsilon}\right) \delta_{k j}+a_{2} \Lambda_{\langle k j\rangle}+a_{3} \Lambda_{i i} \delta_{k j}+o(2), \\
0 & =\frac{\partial \check{\Phi}_{k}}{\partial F_{i \beta}}=\frac{\partial \alpha}{\partial F_{i \beta}} \lambda_{k}+\frac{\partial \beta}{\partial F_{i \beta}} \Lambda_{k}+o(2),
\end{aligned}
$$

para os fluxos. Já é sabido, (4.6) $)_{1}$ e $(4.1)_{1}$, que os multiplicadores de Lagrange $\Lambda_{\varepsilon}, \Lambda_{i j}$ se anulam no equilíbrio. Logo

$$
\hat{p}_{i k}^{o}=\beta \delta_{i k} \quad \text { e } \quad \beta=\frac{1}{3} \hat{p}_{s s}^{o} .
$$


As duas primeiras equações de (5.4), implicam

$$
-\frac{1}{\theta^{2}} \hat{q}_{k}=\left(\frac{\partial \alpha}{\partial \theta}-\left.\frac{5}{3} \varrho_{s s}\right|_{E} \frac{\partial \beta}{\partial \theta}\right) \lambda_{k}+o(2) \quad \text { e } \quad \hat{q}_{k}=\left(a_{1}-\left.\frac{5}{3} \varrho_{s s}\right|_{E} b_{1}\right) \lambda_{k}+o(2) .
$$

Substituindo uma na outra, tem-se

$$
0=\frac{\partial \alpha}{\partial F_{i \beta}}-\left.\frac{5}{3} \varrho_{s s}\right|_{E} \frac{\partial \beta}{\partial F_{i \beta}}
$$

O traço e a parte simetrizada sem traço da equação $(3.3)_{4}$, fornecerão relações de interesse. Ao calcular a simetrização sem traço de cada um dos membros de $(3.3)_{4}$, será obtida a equação

$$
0=-\varrho_{\kappa} \lambda_{\langle i k\rangle}+\Lambda \hat{p}_{\langle i k\rangle}+2 \Lambda_{j\langle i} \hat{p}_{k\rangle j}+2 \hat{p}_{j\langle i k\rangle} \lambda_{j}+\hat{p}_{j j\langle k} \lambda_{i\rangle} .
$$

A parte linear do traço de $(3.3)_{4}$ reduz-se a

$$
\varrho_{\kappa}+\left(c_{\theta}-2\right) \beta=\frac{1}{\theta}\left(3 b_{3}-c_{\theta} b_{1}\right) .
$$

Em (4.6) foi definido o tensor $\hat{S}_{i j}$, mais conhecido como tensor viscoso. A partir deste tensor, é definida a pressão dinâmica $p_{d}=-\frac{1}{3} \hat{S}_{k k}$. É evidente que a pressão dinâmica herda do tensor viscoso a propriedade de se anular no equilíbrio. Por

$$
\hat{p}_{i j}=\left(\beta+p_{d}\right) \delta_{i j}-\hat{S}_{\langle i j\rangle},
$$

e as relações $(5.4)_{3},(5.1)_{2,3},(5.2)_{1}$ tem-se que

$$
p_{d}=\left(3 b_{3}-c_{\theta} b_{1}\right) 2 h_{3} \varrho_{\varepsilon}+o(2), \quad \hat{S}_{\langle i j\rangle}=-\frac{\theta}{2 h_{2}}\left(\varrho_{\kappa}-2 \beta\right) \varrho_{\langle i j\rangle}+o(2) .
$$

Há ainda, mais uma equação a ser obtida usando as representações (4.10) de $\eta$ e $\breve{\Phi}_{k}$, somente até segunda ordem. A saber, a equação c escrita mediante termos de segunda ordem que, pela independência linear dos tensores $\varrho_{\langle k i\rangle}$ e $\delta_{k i}$, implica

$$
\begin{gathered}
\frac{1}{h_{2}}\left(\frac{\partial a_{2}}{\partial F_{i \beta}}-\left.\frac{5}{3} \varrho_{s s}\right|_{E} \frac{\partial b_{2}}{\partial F_{i \beta}}\right)-4 \frac{\partial \beta}{\partial F_{i \beta}}=0 \\
\frac{5}{3} \frac{\partial \beta}{\partial F_{i \beta}}+2 c_{\theta} h_{3}\left(\frac{\partial a_{1}}{\partial F_{i \beta}}-\left.\frac{5}{3} \varrho_{s s}\right|_{E} \frac{\partial b_{1}}{\partial F_{i \beta}}\right)-6 h_{3}\left(\frac{\partial a_{3}}{\partial F_{i \beta}}-\left.\frac{5}{3} \varrho_{s s}\right|_{E} \frac{\partial b_{3}}{\partial F_{i \beta}}\right)=0 .
\end{gathered}
$$

Em resumo, as equações constitutivas para os fluxos são

$$
\begin{aligned}
\hat{q}_{k} & =\frac{A_{1}}{2 h_{1}} \varrho_{i i k}+o(2), \\
\hat{p}_{i k} & =\left(\beta+p_{d}\right) \delta_{i k}-\hat{S}_{\langle i k\rangle}, \\
\hat{p}_{i j k} & =\frac{A_{2}}{2 h_{1}} \varrho_{n n\langle i} \delta_{j\rangle k}+\frac{A_{3}}{2 h_{1}} \varrho_{n n k} \delta_{i j}+o(2), \\
\hat{p}_{i i j k} & =\alpha \delta_{k j}+\left(3 a_{3}-c_{\theta} a_{1}\right) 2 h_{3} \varrho_{\varepsilon} \delta_{k j}+\frac{a_{2}}{2 h_{2}} \varrho_{\langle k j\rangle}+o(2),
\end{aligned}
$$


com

$$
\begin{aligned}
A_{i} & =a_{i}-\left.\frac{5}{3} \varrho_{s s}\right|_{E} b_{i}, & A_{1} & =-\theta^{2}\left(\frac{\partial \alpha}{\partial \theta}-\left.\frac{5}{3} \varrho_{s s}\right|_{E} \frac{\partial \beta}{\partial \theta}\right), \\
0 & =\frac{\partial \alpha}{\partial F_{i \beta}}-\left.\frac{5}{3} \varrho_{s s}\right|_{E} \frac{\partial \beta}{\partial F_{i \beta}}, & p_{d} & =\left(3 b_{3}-c_{\theta} b_{1}\right) 2 h_{3} \varrho_{\varepsilon}+o(2), \\
c_{\theta} & =\left(\frac{\partial \varepsilon}{\partial \theta}\right)^{-1} \frac{\left.\partial \varrho_{s s}\right|_{E}}{\partial \theta}, & \hat{S}_{\langle i j\rangle} & =-\frac{\theta}{2 h_{2}}\left(\varrho_{\kappa}-2 \beta\right) \varrho_{\langle i j\rangle}+o(2), \\
\beta & =\frac{1}{3} \hat{p}_{s s}^{o}, & b_{2} & =\left(\varrho_{\kappa}-2 \beta\right) \theta .
\end{aligned}
$$

\section{Produção de Entropia}

Foi visto na Seção 4 que a produção de entropia $s$ é mínima no estado de equilíbrio. Além da condição (4.1), para que $s$ seja mínima no equilíbrio, é exigido também que

$$
\left.\frac{\partial^{2} s}{\partial X_{A} \partial X_{B}}\right|_{E} \geq 0
$$

onde $X_{A}$ é da forma $\left(\Lambda_{i \beta}^{\varepsilon}, \Lambda_{\varepsilon}, \Lambda_{i j}, \lambda_{j}\right)$.

As funções $\pi_{i j}$ e $\pi_{i i j}$ dependem de $\left(\lambda_{i \beta}, \Lambda, \Lambda_{i j}, \lambda_{j}\right)$ e conseqüentemente, dependem de $\left(\Lambda_{i \beta}^{\varepsilon}, \Lambda_{\varepsilon}, \Lambda_{i j}, \lambda_{j}\right)$. Portanto as representações lineares de $\pi_{i j}$ e $\pi_{i i j}$ são dadas por

$$
\begin{aligned}
\pi_{i j} & =r_{1} \Lambda_{i j}+r_{2} \Lambda_{i j}^{\varepsilon}+r_{3} \Lambda_{\varepsilon} \delta_{i j}+o(2), \\
\pi_{i i j} & =\tau \lambda_{j}+o(2),
\end{aligned}
$$

onde $r_{1}, r_{2}, r_{3}$ e $\tau$ são funções de $\left(F_{i j}, \theta\right)$. Então a expressão $(3.3)_{5}$ para a produção de entropia torna-se

$$
s=\varrho_{\kappa}\left(r_{1} \Lambda_{i j} \Lambda_{i j}+r_{2} \Lambda_{i j}^{\varepsilon} \Lambda_{i j}+r_{3} \Lambda_{\varepsilon} \Lambda_{i i}+\tau \lambda_{j} \lambda_{j}\right)+o(3) .
$$

A inequação (6.1) é agora rescrita como

$$
\left.w \cdot \frac{\partial^{2} s}{\partial X_{A} \partial X_{B}}\right|_{E} w=2 r_{2} \Lambda_{i j} \Lambda_{i j}^{\varepsilon}+2 r_{3} \Lambda_{i i} \Lambda_{\varepsilon}+2 r_{1} \Lambda_{i j} \Lambda_{i j}+2 \tau \lambda_{p} \lambda_{p} \geq 0,
$$

para todo vetor $w=\left(\Lambda_{i j}^{\varepsilon}, \Lambda_{\varepsilon}, \Lambda_{i j}, \lambda_{p}\right)$. Particularizamos os vetores $w$ e obtivemos restrições sobre os coeficientes $r_{1}, r_{2}, r_{3}$ e $\tau$. De fato, fizemos somente $\Lambda_{12}$ não nulo e obtivemos $r_{1} \geq 0$. Fizemos somente $\lambda_{1}$ não nulo e obtivemos $\tau \geq 0$ e por último, tomamos $w$ cujas coordenadas $\lambda_{p}=0$ para $p=1,2,3, \Lambda_{k l}=0$ para $k \neq l$, e concluímos que

$$
2 r_{2} \Lambda_{j j} \Lambda_{i i}^{\varepsilon}+2 r_{3} \Lambda_{i i} \Lambda_{\varepsilon} \geq 0
$$

para todo $\Lambda_{j j}, \Lambda_{i i}^{\varepsilon}$ e $\Lambda_{\varepsilon}$. Fizemos $\Lambda_{\varepsilon}=0$ e obtivemos $r_{2}=0$ e por conseguinte que $r_{3}=0$.

Da invertibilidade de $\pi_{i j}$ na variável $\Lambda_{i j}$, e de $\pi_{i i j}$ na variável $\lambda_{j}$, tem-se

$$
r_{1}>0 \quad \text { e } \tau>0
$$




\section{Concavidade da Densidade de Entropia}

Uma condição suficiente para que o sistema (2.1) e (2.2) seja hiperbólico é $h(\boldsymbol{u})$ ser uma função côncava [10]. Isto ocorrerá se, e somente se

$$
\delta \hat{\boldsymbol{\Lambda}} \cdot \delta \boldsymbol{U}<0 \quad \text { para todas as variações } \delta \hat{\boldsymbol{\Lambda}}, \delta \boldsymbol{U},
$$

onde $\delta \hat{\Lambda}=\left(\delta\left(\varrho_{\kappa} \hat{\lambda}_{s \alpha}\right), \delta \hat{\Lambda}_{i}, \delta \hat{\Lambda}, \delta \hat{\Lambda}_{i j}, \delta \hat{\lambda}_{j}\right)$ e $\delta \boldsymbol{U}=\left(\delta F_{s \alpha}, \delta\left(\varrho v_{i}\right), \delta(\varrho e), \delta u_{i j}, \delta u_{i i j}\right)$.

Por se tratar de uma forma quadrática, e supondo $\dot{\varrho}_{\kappa}=0$ e $\operatorname{det} F>0$, a expressão $\delta \hat{\boldsymbol{\Lambda}} \cdot \delta \boldsymbol{u}$ é posta na forma $\boldsymbol{w} \cdot A \boldsymbol{w}$ onde $\boldsymbol{w}=\left(\delta F_{i j}, \delta \theta, \delta \varrho_{\varepsilon}, \delta v_{k}, \delta \varrho_{\langle k l\rangle}, \delta \varrho_{s s k}\right)$ e $A$ é uma matriz negativa definida composta de blocos e coeficientes $C_{i j r s}, C_{v_{i} v_{k}}, C_{\theta \theta}$.

Da relação de Gibbs (4.3), tira-se

$$
\frac{\partial \eta_{0}}{\partial \theta}=\frac{1}{\theta} \frac{\partial \varepsilon}{\partial \theta} \quad \text { e } \quad \theta \frac{\partial \eta_{0}}{\partial F_{i j}}=\frac{\partial \varepsilon}{\partial F_{i j}}+\frac{1}{\varrho_{\kappa}} \hat{p}_{i j}^{o} .
$$

Pela expressão de $c_{\theta}$, tem-se que

$$
c_{\theta} \frac{\partial \varepsilon}{\partial \theta}=\frac{\left.\partial \varrho_{s s}\right|_{E}}{\partial \theta} .
$$

É sabido que uma matriz negativa definida possui os coeficientes da diagonal negativos. Portanto,

$$
C_{i j r s}<0 \text { para } i=j=r=s, C_{\theta \theta}<0, C_{v_{i} v_{k}}<0 \text { para } i=k, \text { e } h_{1}<0 .
$$

Em particular,

$$
\frac{\partial \hat{p}_{i j}^{0}}{\partial F_{r s}}=\left.\theta C_{i j r s}\right|_{E}<0 \text { para } i=j=r=s \quad \text { e } \quad \frac{\partial \varepsilon}{\partial \theta}=-\left.\frac{\theta^{2}}{\varrho} C_{\theta \theta}\right|_{E}>0 .
$$

A relação $(7.4)_{2}$ juntamente com a $(7.1)_{1}$ implicam que

$$
\frac{\partial \eta_{0}}{\partial \theta}>0
$$

além de mostrar que o calor específico $c_{v}=\frac{\partial \varepsilon}{\partial \theta}$ a volume constante, é positivo.

\section{Conclusão}

Esse trabalho, feito para materiais viscoelásticos, é uma ampliação do trabalho feito em [13] feito para fluidos. Pelo fato do esperado ter ocorrido, ou seja, obter os resultados (5.12), (6.4) e (7.5) com similaridade aos obtidos em [13], ficamos satisfeitos com os resultados. Aqui, a originalidade é, no mínimo, a mesma que em [13], ou seja, com um menor número de variáveis, foram obtidos os resultados em (5.12), (5.13). Uma extensão direta desse trabalho seria usar os termos de ordem 3 para a densidade e fluxos de entropia em (4.10) e uma outra extensão seria encontrar um sistema de equações diferenciais parciais quase-linear para um sólido com condução de calor. 


\section{Apêndice}

A matriz $A$ da seção 7 é igual a

$\left[\begin{array}{cccccc}{\left[C_{i j r s}\right]_{9 \times 9}} & {\left[C_{i j \theta}\right]_{9 \times 1}} & {\left[C_{i j \varrho_{\varepsilon}}\right]_{9 \times 1}} & {\left[C_{i j v_{k}}\right]_{9 \times 3}} & {\left[D_{i j r s}\right]_{9 \times 9}} & {\left[D_{i j k}\right]_{9 \times 3}} \\ {\left[C_{i j \theta}\right]_{1 \times 9}^{T}} & {\left[C_{\theta \theta}\right]_{1 \times 1}} & {\left[C_{\theta \varrho \varepsilon}\right]_{1 \times 1}} & {\left[C_{\theta v_{k}}\right]_{1 \times 3}} & {\left[C_{\theta k l}\right]_{1 \times 9}} & {\left[C_{\theta k}\right]_{1 \times 3}} \\ {\left[C_{i j \varrho_{\varepsilon}}\right]_{1 \times 9}^{T}} & {\left[C_{\theta \varrho_{\varepsilon}}\right]_{1 \times 1}^{T}} & 0 & {\left[-\varrho \frac{2 \varrho_{l l k}}{3 h_{1}}\right]_{1 \times 3}} & {[0]_{1 \times 9}} & {[0]_{1 \times 3}} \\ {\left[C_{i j v_{k}}\right]_{3 \times 9}^{T}} & {\left[C_{\theta v_{i}}\right]_{3 \times 1}^{T}} & {\left[-\varrho \frac{2 \varrho_{l l k}}{3 h_{1}}\right]_{3 \times 1}^{T}} & {\left[C_{v_{i} v_{k}}\right]_{3 \times 3}} & {\left[-\varrho \frac{\varrho_{n n l}}{2 h_{1}} \delta_{i k}\right]_{3 \times 9}} & {\left[\varrho \frac{v_{i} v_{k}}{2 h_{1}}\right]_{3 \times 3}} \\ {\left[D_{i j r s}\right]_{9 \times 9}^{T}} & {\left[C_{\theta k l}\right]_{9 \times 1}^{T}} & {[0]_{9 \times 1}} & {\left[-\varrho \frac{\varrho_{n n l}}{2 h_{1}} \delta_{i k}\right]_{9 \times 3}^{T}} & {[0]_{9 \times 9}} & {\left[\frac{\varrho v_{k}}{2 h_{1}} \delta_{i l}\right]_{9 \times 3}} \\ {\left[D_{i j k}\right]_{3 \times 9}^{T}} & {\left[C_{\theta k}\right]_{3 \times 1}^{T}} & {[0]_{3 \times 1}} & {\left[\varrho \frac{v_{i} v_{k}}{2 h_{1}}\right]_{3 \times 3}} & {\left[\frac{\varrho v_{k}}{2 h_{1}} \delta_{i l}\right]_{3 \times 9}^{T}} & \frac{\varrho}{2 h_{1}} I d_{3 \times 3}\end{array}\right]$,

onde os seus coeficientes desconhecidos são apresentados abaixo:

$$
\begin{aligned}
C_{i j r s}= & \frac{1}{\theta} \frac{\partial \hat{p}_{i j}^{0}}{\partial F_{r s}}+\varrho_{\kappa}\left(2 \frac{\partial h_{3}}{\partial F_{r s}} \varrho_{\varepsilon} \delta_{i j}-\frac{1}{2 h_{2}^{2}} \frac{\partial h_{2}}{\partial F_{r s}} \varrho_{\langle i j\rangle}\right)-2 \frac{\varepsilon \varrho_{\kappa}}{\operatorname{det} F} F_{s r}^{-1} \varrho_{\varepsilon}\left(\frac{\partial c_{\theta}}{\partial F_{i j}} h_{3}+\frac{\partial h_{3}}{\partial F_{i j}} c_{\theta}\right) \\
& -2 \varrho \frac{\partial \varepsilon}{\partial F_{r s}} \varrho_{\varepsilon}\left(\frac{\partial c_{\theta}}{\partial F_{i j}} h_{3}+\frac{\partial h_{3}}{\partial F_{i j}} c_{\theta}\right)-\frac{\varrho}{3 h_{1}^{2}} v_{k} \varrho_{l l k} \frac{\partial h_{1}}{\partial F_{r s}} \frac{\left.\partial \varrho_{n n}\right|_{E}}{\partial F_{i j}} \\
& +v_{l}\left\{-\frac{v_{k} v_{k}}{h_{1}^{2}} \varrho_{n n l} \frac{\partial h_{1}}{\partial F_{r s}}-v_{k}\left(2 \varrho_{\varepsilon} \frac{\partial h_{3}}{\partial F_{r s}}-\frac{\varrho_{\langle k l\rangle}}{2 h_{2}^{2}} \frac{\partial h_{2}}{\partial F_{r s}}\right)+v_{l} \varrho_{\varepsilon}\left(h_{3} \frac{\partial c_{\theta}}{\partial F_{r s}}+c_{\theta} \frac{\partial h_{3}}{\partial F_{r s}}\right)\right. \\
& \left.-\frac{1}{3 h_{1}} \varrho_{k k l} \frac{\left.\partial \varrho_{n n}\right|_{E}}{\partial F_{r s}}\right\} \frac{\varrho_{\kappa}}{\operatorname{det} F} F_{j i}^{-1}, \\
C_{i j \theta}= & -\frac{\varepsilon \varrho_{\kappa}}{\operatorname{det} F} F_{j i}^{-1} \varrho_{\varepsilon}\left(\frac{\partial c_{\theta}}{\partial \theta} h_{3}+\frac{\partial h_{3}}{\partial \theta} c_{\theta}\right)+\frac{1}{2 \theta^{2}}\left[\theta \frac{\partial \hat{p}_{i j}^{0}}{\partial \theta}-\hat{p}_{i j}^{0}-\varrho_{\frac{\partial \varepsilon}{\partial F_{i j}}}-\frac{\varepsilon \varrho_{\kappa}}{\operatorname{det} F} F_{j i}^{-1}\right] \\
& +\varrho_{\kappa}\left(\frac{\partial h_{3}}{\partial \theta} \varrho_{\varepsilon} \delta_{i j}-\frac{1}{4 h_{2}^{2}} \frac{\partial h_{2}}{\partial \theta} \varrho_{\langle i j\rangle}\right)-\varrho \frac{\partial \varepsilon}{\partial \theta} \varrho_{\varepsilon}\left(\frac{\partial c_{\theta}}{\partial F_{i j}} h_{3}+\frac{\partial h_{3}}{\partial F_{i j}} c_{\theta}\right) \\
& -\varrho \frac{\partial \varepsilon}{\partial F_{i j}} \varrho_{\varepsilon}\left(\frac{\partial c_{\theta}}{\partial \theta} h_{3}+\frac{\partial h_{3}}{\partial \theta} c_{\theta}\right)-\frac{\varrho}{3 h_{1}^{2}} v_{k} \varrho_{l l k} \frac{\partial h_{1}}{\partial F_{i j}} \frac{\left.\partial \varrho_{n n}\right|_{E}}{\partial \theta} \\
& +\frac{v_{l}}{2}\left\{-\frac{v_{k} v_{k}}{h_{1}^{2}} \varrho_{n n l} \frac{\partial h_{1}}{\partial \theta}-2 \varrho_{\varepsilon} \frac{\partial h_{3}}{\partial \theta} v_{l}+\frac{v_{k}}{2 h_{2}^{2}} \varrho_{\langle k l\rangle} \frac{\partial h_{2}}{\partial \theta}+v_{l} \varrho_{\varepsilon}\left(h_{3} \frac{\partial c_{\theta}}{\partial \theta}+c_{\theta} \frac{\partial h_{3}}{\partial \theta}\right)+\frac{v_{l}}{\theta^{2}}\right. \\
& \left.-\frac{1}{3 h_{1}} \varrho_{k k l} \frac{\partial \varrho_{n n} \mid{ }_{E}}{\partial \theta}\right\} \frac{\varrho_{\kappa}}{\operatorname{det} F} F_{j i}^{-1}, \\
C_{i j} \varrho_{\varepsilon} & =\varrho_{\kappa} h_{3} \delta_{i j}-\varrho \frac{\partial \varepsilon}{\partial F_{i j}} c_{\theta} h_{3}-\left(\varepsilon c_{\theta} h_{3}+2 v_{s} v_{s} h_{3}+2 c_{\theta} h_{3}+\frac{v_{l}}{6 h_{1}} \varrho_{k k l}\right) \frac{\varrho_{\kappa}}{\operatorname{det} F} F_{j i}^{-1} \\
& \\
& \\
&
\end{aligned}
$$




$$
\begin{aligned}
C_{i j v_{k}}= & \frac{1}{2}\left[v_{k}\left(2 c_{\theta} \varrho_{\varepsilon} h_{3}-\frac{1}{\theta}\right)-\frac{v_{l}}{h_{2}} \varrho_{\langle k l\rangle}-4 h_{3} v_{k} \varrho_{\varepsilon}+\frac{v_{l} v_{l}}{2 h_{1}} \varrho_{n n k}+\frac{v_{k} v_{l}}{h_{1}} \varrho_{n n l}\right. \\
& \left.-\frac{5}{6} \frac{\left.\varrho_{n n}\right|_{E}}{h_{1}} \varrho_{l l k}\right] \frac{\varrho_{k}}{\operatorname{det} F} F_{j i}^{-1}-\varrho \frac{v_{k} v_{l}}{2 h_{1}^{2}} \varrho_{n n l} \frac{\partial h_{1}}{\partial F_{i j}}-\frac{4}{6} \frac{\varrho}{h_{1}} \varrho_{l l k} \frac{\left.\partial \varrho_{s s}\right|_{E}}{\partial F_{i j}}, \\
D_{i j r s}= & \frac{\varrho_{\kappa}}{4 h_{2}} \delta_{r i} \delta_{s j}-\frac{1}{2}\left(v_{r} v_{s} \frac{1}{2 h_{2}}+\frac{v_{s}}{h_{1}} \varrho_{l l r}\right) \frac{\varrho_{\kappa}}{\operatorname{det} F} F_{j i}^{-1}-\frac{\varrho}{2 h_{1}^{2}} v_{r} \varrho_{l l s} \frac{\partial h_{1}}{\partial F_{i j}}, \\
D_{i j k}= & \left(\varrho_{l l k}-2 v_{l} \varrho_{l k}-v_{s} \varrho_{l l}+2 v_{l} v_{l} v_{k}\right) \frac{1}{4 h_{1}} \frac{\varrho_{\kappa}}{\operatorname{det} F} F_{j i}^{-1}+\frac{\varrho}{6 h_{1}} v_{k} \frac{\left.\partial \varrho_{n n}\right|_{E}}{\partial F_{i j}}-\frac{\varrho}{4 h_{1}^{2}} \varrho_{l l k} \frac{\partial h_{1}}{\partial F_{i j}}, \\
C_{\theta \theta}= & -\frac{\varrho}{\theta^{2}} \frac{\partial \varepsilon}{\partial \theta}-2 \varrho \frac{\partial \varepsilon}{\partial \theta} \varrho_{\varepsilon}\left(\frac{\partial c_{\theta}}{\partial \theta} h_{3}+\frac{\partial h_{3}}{\partial \theta} c_{\theta}\right)-\frac{\varrho}{3 h_{1}^{2}} v_{r} \varrho_{l l r} \frac{\partial h_{1}}{\partial \theta} \frac{\left.\partial \varrho_{s s}\right|_{E}}{\partial \theta}, \\
C_{\theta \varrho_{\epsilon}}= & -\varrho \frac{\partial \varepsilon}{\partial \theta} c_{\theta} h_{3}, \\
C_{\theta v_{k}}= & -\varrho \frac{v k}{2 h_{l}^{2}} \varrho_{n n l} \frac{\partial h_{1}}{\partial \theta}-\frac{2}{3} \frac{\varrho}{h_{1}} \varrho_{n n k} \frac{\left.\partial \varrho_{n n}\right|_{E}}{\partial \theta}, \\
C_{\theta k l}= & -\frac{\varrho}{2 h_{1}^{2}} v_{k} \varrho_{n n l} \frac{\partial h_{1}}{\partial \theta}, \\
C_{\theta k}= & \frac{\varrho}{6 h_{1}} v_{k} \frac{\partial \varrho_{s s} \mid E}{\partial \theta}-\frac{\varrho}{4 h_{1}^{2}} \varrho_{l l k} \frac{\partial h_{1}}{\partial \theta}, \\
C_{v_{i} v_{k}}= & {\left[\frac{\varrho}{\theta}+2 \varrho h_{3} \varrho_{\varepsilon}\left(c_{\theta}+2\right)\right] \delta_{i k}+\frac{\varrho}{2 h_{2}} \varrho_{\langle i k\rangle} . }
\end{aligned}
$$

\section{Referências}

[1] G.M. Kremer, Extended thermodynamics of ideal gases with 14 fields, Ann. Inst. Henri Poincaré, 45 (1986), 419-440.

[2] G.M. Kremer, Extended thermodynamics of non-ideal gases, Physica, 144A (1987), 156-178.

[3] G.M. Kremer, Extended thermodynamics of molecular ideal gases, Continuum Mech. Thermodyn., 1 (1989), 21-45.

[4] G.M. Kremer, C. Beevers, Extended thermodynamics of dense gases, Lecture Note in Physics, 199 (1984), 429-436.

[5] I-Shih Liu, Method of Lagrange multipliers for exploitation of the entropy principle, Arch. Rational Mech. Anal., 46 (1972), 131-148.

[6] I-Shih Liu, An extended field theory of viscoelastic materials, Int. J. Engng Sci., 26 (1988), 331-342.

[7] I-Shih Liu, Extended thermodynamics of viscoelastic materials, Continuum Mech. Thermodyn, 1 (1989), 143-164.

[8] I-ShihLiu, Extended thermodynamics of viscoelasticity, Proceedings of the $5^{\text {th }}$ Bilateral Polish-Italian Meeting, Thermodynamics and kinetic theory. World Scientific Publishing, Singapore, (1990) 93-106 .

[9] I-Shih Liu, "Continuum Mechanics", Springer-Verlag Berlin Heidelberg New York (2002). 
[10] I-Shih Liu, G.M. Kremer, Hyperbolic system of field equations for viscous fluids, Mat. Aplic. Comp., 9 (1990), 123-135.

[11] I-Shih Liu, I. Müller, Extended thermodynamics of classical and degenerate gases, Arch. Rational Mech. Anal., 83 (1983), 285-332.

[12] I. Müller, T. Ruggeri, "Rational Extended Thermodynamics", Second Edition, Springer-Verlag, New York (1998).

[13] A. Vignatti, I-Shih Liu, Termodinâmica estendida de fluidos viscosos com condução de calor, TEMA, SBMAC, numero 2 (2006), 381-390.

[14] R. Resnick, D. Halliday, "Física", v2 $4^{a}$ ed., Livros Técnicos e Científicos Editora S.A. (1984).

[15] T. Ruggeri, Galilean invariance and entropy principle for systems of balance laws, Continuum Mech. Thermodyn., 1 (1989), 3-20. 